\title{
Osteophyte formation involves PAR2
}

\section{ff}

fewer Par $2^{-}$

mice developed osteophytes ... compared with control mice

5

Osteophytes, or bony spurs, are bone outgrowths that form early in osteoarthritis (OA) in an attempt to compensate for joint damage. Huesa et al. now show that proteinase-activated receptor 2 (PAR2) is involved in this process, thereby expanding the known roles of PAR2 in OA beyond cartilage degradation and osteosclerosis.

PAR2, a G protein-coupled receptor, might be involved in $\mathrm{OA}$ through its role in modulating the expression of metalloproteases. Systemic inactivation of Par2 in mice (Par2 ${ }^{-/}$mice) was previously found to slow OA progression.

"In this study, we have integrated a cross-disciplinary range of techniques to allow an holistic approach to elucidating early joint changes in OA," states John Lockhart, co-corresponding author on this paper. Micro-CT showed that fewer $\mathrm{Par}^{-/}$mice developed osteophytes 4 weeks after induction of $\mathrm{OA}$ through destabilization of the medial meniscus compared with control mice; when osteophytes developed, they were smaller and had a greater

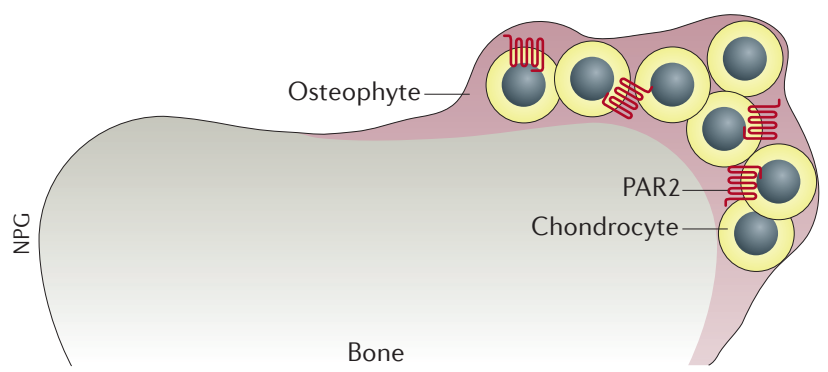

mineral density. Intra-articular injection of human PAR2-expressing adenoviruses reversed the phenotype of $\mathrm{Par}^{-/}$mice to control levels, confirming that Par2 deletion is the causative factor. Histological analysis of the osteophytes in control mice revealed the presence of proliferative and hypertrophic chondrocytes that expressed PAR2. Given that PAR2 is not normally expressed in cartilage, the authors concluded that expression of PAR2 in osteophytes is pathological. Carl Goodyear (co-corresponding author) also explains that they used dynamic weight-bearing as a surrogate indicator of pain, which is a novel approach in OA models. $\mathrm{Par}^{-/-}$mice placed more weight on the affected limb than controls, suggesting impaired nociception.

Taken together, these results demonstrate that PAR2 is not only involved in OA progression, but also regulates early osteophyte formation via its presence in chondrocytes. Moving forward, the authors will focus on the role of osteophyte formation in $\mathrm{OA}$, a process that is not yet well understood. "Our work with PAR2 provides a means by which this process can be interrogated," claims Lockhart.

Liesbet Lieben, Associate Editor Nature Reviews Disease Primers

ORIGINAL ARTICLE Huesa, C. et al.

Proteinase-activated receptor 2 modulates OA-related pain, cartilage and bone pathology. Ann. Rheum. Dis. http://dx.doi.org/10.1136/ annrheumdis-2015-208268 